\title{
Simultaneous identification and typing of multi- drug-resistant Mycobacterium tuberculosis isolates by analysis of pncA and rpoB
}

\author{
T. J. BROWN, Ö. TANSEL* and G. L. FRENCH \\ Department of Microbiology, St. Thomas' Hospital, Lambeth Palace Road, London SE1 7EH and *Kocaeli \\ Unversitesi Tip Fakultesi, Infeksiyon Hastaliklari ve Klinik Mikrobiyoloji ABD, Sopali Ciftligi, Derince, 41900 \\ Izmit, Turkey
}

\begin{abstract}
In Mycobacterium tuberculosis there is a strong correlation between in-vitro resistance to rifampicin (RIF) and pyrazinamide (PZA) and mutations in rpoB and pncA, respectively. Approximately 50 mutations associated with resistance have been reported for $r p o B$ and 70 for $p n c A$, and, theoretically, many more are possible. Therefore, the identification of $r p o B$ and $p n c A$ mutations in $M$. tuberculosis might be used for the simultaneous determination of resistance and for typing multi-drug-resistant (MRD) strains during possible outbreaks. The present study examined four sensitive and six MDR isolates of $M$. tuberculosis from Turkey and eight isolates from a nosocomial MDR tuberculosis (TB) outbreak in the UK. Gene mutations were identified by the Innogenetics LiPA rpoB assay or automated sequencing, or both. All the sensitive isolates had $r p o B$ and $p n c A$ wild-type genotypes, whereas all the RIF- and PZA-resistant isolates had $r p o B$ and $p n c A$ mutations. All four mutations seen in $r p o B$, but none of the six in pncA, had been reported previously. The $r p o B$ and $p n c A$ mutations seen in the Turkish isolates defined six distinct genotypes amongst the six MDR isolates, while standard IS6110 typing discriminated only four. All isolates from the single strain MDRTB outbreak had identical genotypes. Rapid genotyping was performed on the sputum from a patient who presented 2 years after the initial MDR-TB outbreak and this showed $r p o B$ and $p n c A$ genotypes identical to the other outbreak isolates. This result was available within $36 \mathrm{~h}$. The analysis of $r p o B$ and $p n c A$ is a rapid and practical means of simultaneously identifying and typing MDR isolates of M. tuberculosis.
\end{abstract}

\section{Introduction}

A combination of four drugs, isoniazid (INH), rifampicin (RIF), pyrazinamide (PZA) and ethambutol (EMB), with or without streptomycin (STR), is recommended for the standard or first-line treatment of tuberculosis (TB) [1]. Antibiotic resistance has emerged to each of these agents, as has multi-drug resistance (MDR-TB) where the isolate is resistant to at least INH and RIF [2]. The rapid identification of MDR-TB is essential for proper patient management $[1,3]$.

In Mycobacterium tuberculosis, resistance to these first-line drugs is usually associated with mutations in

Received 24 June 1999; revised version accepted 22 Oct. 1999.

Corresponding author: Dr T. J. Brown (e-mail: timothy.brown@kcl.ac.uk). specific genes. EMB resistance is associated with mutations in $e m b B$ in $47-69 \%$ of isolates [4, 5]. Resistance to INH has been associated with a limited number of mutations in $k a t G$ and $\operatorname{inh} A$ [6-8] in up to $75 \%$ of cases [8]. Mutations associated with resistance to RIF are located within a 69-bp region of rpoB [6] in $94-98 \%$ of isolates [9-14]. More recently, mutations within $p n c A$ have been seen in isolates resistant to PZA [15]. PncA mutations, which are distributed throughout the gene, have been reported in $72-100 \%$ of PZA-resistant isolates [15-18]. The high correlation between mutations in $r p o B$ and pncA and phenotypic drug resistance makes these genes attractive targets for the rapid determination of antibiotic susceptibilities of M. tuberculosis. The detection of mutations within $r p o B$ may be used for the presumptive identification of MDR-TB, as MDR strains are usually rifampicinresistant. The relatively small size of $p n c A$ and the limited region of interest within $r$ ro $B$ make the routine analysis of these loci practical. Furthermore, the wide 
range of reported and potential variations within these genes suggests that $r p o B$ and $p n c A$ genotyping might also be used as a rapid method of typing MDR-TB isolates for epidemiological purposes.

To test whether the rapid genetic analysis of $r p o B$ and $p n c A$ can be used for the simultaneous detection of resistance and for typing resistant isolates, the present study analysed these genes in 10 epidemiologically unconnected M. tuberculosis isolates from Istanbul, Turkey, where both primary and acquired resistance is common [19], and isolates from a single strain nosocomial outbreak of MDR-TB in London. In-vitro susceptibilities to RIF and PZA were compared with those predicted by sequence mutations, and the genotypes produced by this method were compared with standard IS6110 typing.

\section{Materials and methods}

\section{Organisms}

Five PZA-resistant and five PZA-sensitive distinct clinical isolates of $M$. tuberculosis complex were obtained from an Istanbul laboratory. Another seven isolates were collected from an outbreak of MDR-TB [20]; four serial isolates were collected over an 18month period from one of these patients and one further isolate was from an epidemiologically related patient who presented 24 months later. A sputum specimen from this final patient was analysed directly after being concentrated [21] and the pellet washed. Organisms were identified by standard methods [22]. INH, RIF and PZA susceptibilities were determined by the Bactec radiometric-based culture method (BectonDickinson, Oxford) according to the manufacturer's standard protocols. PZA susceptibility was also determined by detection of pyrazinamidase activity [23].

\section{Extraction of DNA}

Cells cultured on Lowenstein-Jensen slopes were suspended in a buffer containing $10 \mathrm{~mm}$ Tris, $1 \mathrm{mM}$ EDTA and Triton X100 1\% at pH 8.0. Suspensions were heated to $100^{\circ} \mathrm{C}$ for $30 \mathrm{~min}$, vortex mixed and centrifuged briefly at $13500 \mathrm{rpm}$.

\section{PCR and sequence analysis of pncA}

A PCR product of 599 bp was amplified with primers pncalu (5'-GCCGACGTTGTAAAACGACGGCCAGT GAATTCCAGATGCGGGCGTTGATCATCGTC) and pncA2r (5'-GCCAGGAAACAGCTATGACCGAATT CTGTTCAGGAGCTGCAAACCAACTCG), which were designed by using a published pncA sequence (GenBank MTU59967) and adding universal and reverse primer sequences to the $5^{\prime}$ ends to facilitate sequencing. The amplification product was sequenced with 5'-fluorescein-labelled universal and reverse primers (Pharmacia, St Albans), a Thermo-sequenase cycle sequencing kit (Amersham, Little Chalfont) and an ALF DNA sequencer (Pharmacia). The sequence of each isolate was compared with that of the PZAsensitive wild-type (WT) sequence (GenBank MTU59967). One specimen in this study was analysed by a nested PCR, in which primers P1 (GTCGGTCATGTTCGCGATCG) and P6 (GCT TTGCGGCGAGCGCTCCA) [15] were used in the additional outer PCR.

\section{Analysis of rpoB}

An 80-nucleotide base region of $r p o B$ was analysed and compared with the WT genotype (GenBank L27989) with the Inno-LiPA Rif TB kit (Innogenetics, Zwijnaarde, Belgium) following the manufacturer's protocol. Briefly, this consisted of a nested PCR with biotin-labelled primers; the resultant amplicon was hybridised to an array of oligonucleotides immobilised on a nitrocellulose membrane. Hybrids containing biotin were visualised colorimetrically. Hybridisation with the five Innogenetics WT oligonucleotides (S1S5) indicated that the $r p o B$ was wild-type and the organism was sensitive to RIF; failure to hybridise with one or more of these oligonucleotides indicated a mutant $r p o B$ and, therefore, resistance to RIF was deduced. Four commonly seen specific mutations were identified by hybridisation to one of four mutant oligonucleotides, R1-R4. Other mutants were identified by amplification of a 260-bp region of $r p o B$ with the primers TR1 (5'-TACGGTCGGCGAGCTGATCC) and TR2 (5'-TACGGCGTTTGCATGAACC) [14] and sequencing the product with 5 'fluorescein-labelled TR1 and TR2 in a Thermo-sequenase cycle sequencing kit and an ALF DNA sequencer, as before. The sequence of each isolate was compared with the RIFsensitive WT sequence (GenBank L27989).

\section{DNA typing of M. tuberculosis strains}

DNA was extracted for restriction fragment-length polymorphism (RFLP) analysis with proteinase $\mathrm{K}$ (Boehringer-Mannheim, Lewes) and lysozyme (Sigma), followed by phenol:chloroform extraction. Restriction digests were prepared according to standardised methodology [24] with the restriction endonuclease $P v u$ II. Fragments were separated in an agarose $1.5 \%$ gel and capillary-blotted on to nylon membrane. A digoxigenin-labelled IS6110 probe was generated by PCR with $0.4 \mu \mathrm{M}$ primer 41 and primer 43 [25], $40 \mu \mathrm{M}$ dNTP and $8 \mu \mathrm{M}$ DIG-dUTP. Hybridisation was detected as described previously [26].

\section{Results}

\section{pncA}

The five phenotypically PZA-sensitive Turkish isolates were all genotypically WT and the five phenotypically PZA-resistant isolates were all genotypically mutant. 
All five resistant isolates had different $p n c A$ mutations, as shown in Table 1. Isolate 985040 contained two mutations, one resulting in an amino-acid change when translated and a silent mutation. All other mutations in these Turkish isolates resulted in amino-acid changes when translated. All eight isolates of the London PZAresistant outbreak strain and the four serial isolates had the same pncA mutation, which was different from those seen in the Turkish isolates (Table 1).

\section{гроB}

The four phenotypically RIF-sensitive Turkish isolates had WT rроB genes. Mutations were detected in $r p o B$ in all six Turkish RIF-resistant isolates. Isolate 1028448 was initially designated phenotypically sensitive to RIF but was shown to contain a mutation. On re-testing, this isolate was found to be phenotypically resistant. Four different $r p o B$ mutations were seen amongst these six resistant isolates as shown in Table 1. Mutations in isolates 1032166, 985040, 1017379 and 1015307 were detected with the LiPA assay by hybridisation to R5, $\mathrm{R} 2$, R5 and R2 respectively. Mutations in isolates 1028448 and 992048 were determined by sequencing. Isolate 1015307 was shown to contain a mixed population of $M$. tuberculosis: a WT and a mutant. All eight of the London RIF-resistant outbreak isolates and the serial isolates contained the same mutation (Table 1).

\section{IS6110 typing}

The IS6110-probed restriction patterns for the Turkish isolates investigated in this study are shown in Fig. 1. The four fully sensitive Turkish isolates each had different IS6110 restriction patterns, demonstrating that these WT strains were not linked. Two of the Turkish MDR-TB isolates, 992048 and 1032166, had similar IS6110 typing patterns and resembled that of the WT isolate 1010968. The more intense signal seen in the profile of isolate 1032166 showed an additional fragment not visible in isolates 1010968 and 992048. The other three Turkish MDR-TB isolates had different IS6110 restriction patterns. All the outbreak isolates were indistinguishable by IS6110 typing as previously reported [20] and gave a five band pattern.

\section{rpoB/pncA genotyping}

The nucleotide variations seen within the $r p o B$ and $p n c A$ sequences of the RIF- and PZA-resistant isolates could be used to genotype them. In this study, all the five Turkish MDR-TB isolates had different $r p o B$ or pncA sequences, or both, including the two MDR-TB isolates with similar IS6110 patterns. Thus $r p o B / p n c A$ genotyping discriminated five genotypes amongst the five MDR-TB isolates compared with the four seen with IS6110 typing (Fig. 1). All eight of the London MDR-TB outbreak isolates contained the same

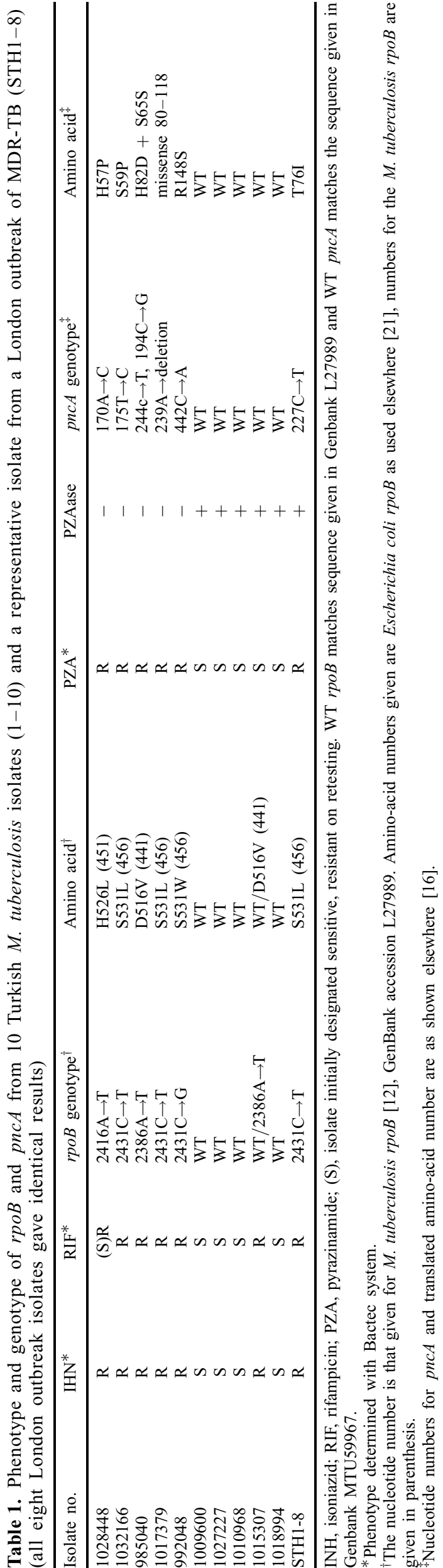




\begin{tabular}{|c|c|c|}
\hline size marker & 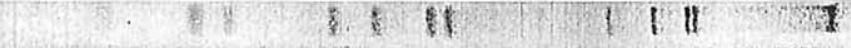 & $r p o B / p n c A$ genotype \\
\hline 1028448 & 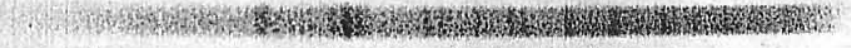 & $2416 \mathrm{~A} \rightarrow \mathrm{T} / 170 \mathrm{~A} \rightarrow \mathrm{C}$ \\
\hline 1032166 & 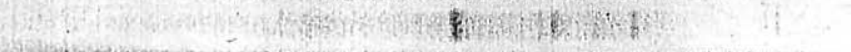 & $2431 \mathrm{C} \rightarrow \mathrm{T} / 175 \mathrm{~T} \rightarrow \mathrm{C}$ \\
\hline 985040 & 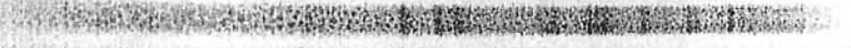 & $2386 \mathrm{~A} \rightarrow \mathrm{T} / 244 \mathrm{C} \rightarrow \mathrm{T}, 194 \mathrm{C} \rightarrow \mathrm{G}$ \\
\hline 1017379 & 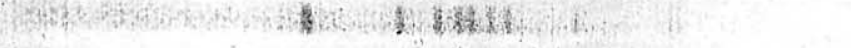 & $2431 \mathrm{C} \rightarrow \mathrm{T} / 239 \mathrm{~A} \rightarrow$ deletion \\
\hline 992048 & & $2431 \mathrm{C} \rightarrow \mathrm{G} / 442 \mathrm{C} \rightarrow \mathrm{A}$ \\
\hline 1009600 & 5. & WT / WT \\
\hline 1027227 & 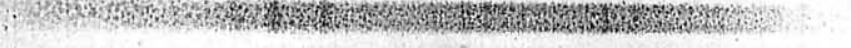 & WT / WT \\
\hline 1010968 & $\cdot \quad+13$ & WT / WT \\
\hline 1015307 & 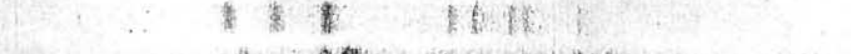 & $(\mathrm{WT} / 2386 \mathrm{~A} \rightarrow \mathrm{T}) / \mathrm{WT}$ \\
\hline 1018994 & $\therefore \quad 4.45$ a & $\mathrm{WT} / \mathrm{WT}$ \\
\hline
\end{tabular}

Fig. 1. IS6110 typing patterns seen with 10 Turkish isolates. Size marker lane contains digoxigenin-labelled markers (Boehringer-Mannheim) of (from left to right), 125, 564, 831, 947, 1375, 1584, 1904, 2027, 3530, 4268, 4973, 5148, $21226 \mathrm{bp}$.

rpoB/pncA mutation combination, as did the serial isolates.

\section{Discussion}

In this study, the Turkish and UK MDR M. tuberculosis isolates contained mutations in rpoB and pncA that were associated with phenotypic resistance to RIF and PZA, respectively, whereas none of the RIF- or PZAsensitive isolates examined contained mutations. These results confirm those of other workers reported from other geographical locations [9-18].

The determination of genotype to predict phenotypic resistance to RIF and PZA is much more rapid than traditional culture methods, taking hours rather than weeks. Standard methods of pyrazinamide susceptibility testing can be unreliable $[27,28]$ and are usefully supplemented by testing for pyrazinamidase production [29]. However, pncA genotyping can be performed several weeks earlier than pyrazinamidase production, as it can be done directly from a primary isolation slope and is probably more reliable.

Previous studies have described 71 different mutations in pncA associated with PZA resistance [15-18]. These consisted of 55 substitutions, 12 deletions and six insertions. Two previously described pncA mutations, consisting of a base change at the -11 position [18] and a deletion of 1-11 [15], would not be detected by the methodology described in this report, which uses primers based on the first and last 20 bases of the pncA coding region. None of the six pncA missense mutations seen in the present study, consisting of five substitutions and one deletion, have been described previously. One of the resistant isolates with a missense mutation also contained a silent pncA mutation. A silent mutation in rроB has been seen before [30], but has not been reported previously in pncA. The existence of silent mutations demonstrates the value of identifying all nucleotide base changes in order to predict amino-acid sequence and hence antibiotic susceptibility.

The five Turkish PZA-resistant isolates in the present study had five different $p n c A$ genotypes, but only four IS6110 types, suggesting that, for resistant isolates, pncA typing may be more useful for discriminating potentially related strains than IS6110 typing. Only four of the approximately $70 p n c A$ genotypes reported so far have been seen in more than one geographical location, and each of these occurred in two locations only [15-18]. There are 1235 possible single base mutations in pncA that can result in an amino-acid change or a stop codon. There are even more possible variants if the less common mutations are included, such as multiple base changes, deletions, insertions and mutations outside the $p n c A$ coding region. If these mutations are stable, the characterisation of $p n c A$ could provide a powerful tool for studying the epidemiology of MDR-TB.

Fifty mutations have been reported in $r p o B$ in isolates from various geographical locations [9-14]. Some mutations occur much more frequently than others. For example S531L, which was seen in two isolates in the present study, is common [9-14], whereas $\mathrm{N} 518 \mathrm{H}$ has been reported only once [11]. The reason for this variation in frequency of mutant genotypes is not clear. All four mutations in $r p o B$ seen in this study have been reported previously. One of the cultures yielded two rpoB genotypes: the mutant $2386 \mathrm{~A} \rightarrow \mathrm{T}$ and a WT. It is thought that resistant M. tuberculosis cells may appear in WT populations by spontaneous mutation; in the presence of RIF these cells are selected, shifting the population from predominantly WT to a predominantly resistant phenotype. This mixed culture may have been from a patient where this change was taking place.

The six Turkish RIF-resistant isolates in the present study could be separated into four rроB genotypes and five IS6110 types. Although, in this sample, IS6110 
typing gave a greater discrimination than the $r p o B$ genotypes, the two isolates with similar IS6110 types had different $r$ rрoB genotypes.

When both pncA and rpoB sequence analysis were used to type the six Turkish MDR-TB isolates, six genotypes were discriminated, compared with only five by IS6110 typing. Although the present study examined only a small number of resistant strains, the ability of $r p o B / p n c A$ typing to distinguish all of them is striking. Furthermore, the large number of reported and theoretical mutations associated with resistance suggests that analysis of these genes would be useful for the epidemiology of resistant tuberculosis.

The rpoB/pncA genotype was identical in isolates from the eight epidemiologically linked patients [20], as were serial isolates obtained from one patient over an 18-month period. Also, one of the cases was identified as being positive for MDR-TB 2 years after the initial outbreak. It has been shown that IS6110 probing is a stable typing tool [31]. These results suggest that the genotyping used in the present study is also stable. This is shown during patient-to-patient transmission (the outbreak isolates), during infection (the isolate cultured subsequent to the main outbreak) and during active disease (the serial isolates).

The use of these gene mutations for the simultaneous detection of antimicrobial resistance and for rapid typing is particularly useful for determining whether two or more hospital isolates of MDR-TB represent an outbreak, as described above. In this setting, this genotyping technique may be more useful than IS6110 typing, because it is rapid, the interpretation of the results is simple, the method is highly discriminatory and it can be performed directly from specimens without waiting for culture. Of course, rpoB/pncA typing does not have the universal application of IS6110 typing, as it can be applied only to resistant isolates of M. tuberculosis, different strains may develop the same rpoB/pncA mutations, and the technique cannot identify relatedness between sensitive and resistant isolates of the same strain.

The characterisation of $r p o B$ and $p n c A$ provides a practical and rapid prediction of $M$. tuberculosis susceptibility to RIF and PZA, and can be used simultaneously for outbreak typing of resistant strains. An obvious limitation of this method is that automated sequencing is required for the analysis of $p n c A$ and for mutations in $r p o B$ for which there are no LiPA probes. However, for laboratories with the necessary equipment and expertise, analysis of $r p o B$ and $p n c A$ can be done routinely and rapidly on selected specimens and isolates. Simpler methods for detecting genotype variations in isolates or directly in clinical specimens would be powerful clinical tools for the diagnosis and management of MDR-TB.
We thank Dr K. Köksalan and Duzen laboratories for providing the Turkish isolates examined in this study.

\section{References}

1. Joint Tuberculosis Committee of the British Thoracic Society. Chemotherapy and management of tuberculosis in the United Kingdom: recommendations 1998. Thorax 1998; 53: 536-548.

2. WHO/IUATLD Global Project. Anti-tuberculosis drug-resistance in the world. Geneva, Switzerland, WHO. 1997.

3. American Thoracic Society. Treatment of tuberculosis and tuberculosis infection in adults and children. Am J Respir Crit Care Med 1994; 149: 1359-1374.

4. Sreevatsan S, Stockbauer KE, Pan X et al. Ethambutol resistance in Mycobacterium tuberculosis: critical role of embB mutations. Antimicrob Agents Chemother 1997; 41: 1677-1681.

5. Telenti A, Philipp WJ, Sreevatsan S et al. The emb operon, a unique gene cluster of Mycobacterium tuberculosis involved in resistance to ethambutol. Nat Med 1997; 3: 567-570.

6. Blanchard JS. Molecular mechanisms of drug resistance in Mycobacterium tuberculosis. Annu Rev Biochem 1996; 65: 215-239.

7. Haas WH, Schilke K, Brand $\mathrm{J}$ et al. Molecular analysis of kat $G$ gene mutations in strains of Mycobacterium tuberculosis complex from Africa. Antimicrob Agents Chemother 1997; 41: $1601-1603$.

8. Musser JM, Kapur V, Williams DL, Kreiswirth BN, van Soolingen D, van Embden JDA. Characterization of the catalase-peroxidase gene (kat $\mathrm{G}$ ) and inhA locus in isoniazidresistant and -susceptible strains of Mycobacterium tuberculosis by automated DNA sequencing: restricted array of mutations associated with drug resistance. J Infect Dis 1996; 173: $196-202$.

9. Cooksey RC, Morlock GP, Glickman S, Crawford JT. Evaluation of a Line Probe Assay Kit for characterization of rрoB mutations in rifampicin-resistant Mycobacterium tuberculosis isolates from New York City. J Clin Microbiol 1997; 35: $1281-1283$.

10. De Beenhouwer H, Lhiang Z, Jannes G et al. Rapid detection of rifampicin resistance in sputum and biopsy specimens from tuberculosis patients by PCR and line probe assay. Tubercle Lung Dis 1995; 76: 425-430.

11. Kapur V, Li L-L, Iordanescu $\mathrm{S}$ et al Characterization by automated DNA sequencing of mutations in the gene (rpoB) encoding the RNA polymerase $\beta$ subunit in rifampicin resistant Mycobacterium tuberculosis strains from New York City and Texas. J Clin Microbiol 1994; 32: 1095-1098.

12. Miller LP, Crawford JT, Shinnick TM. The rpoB gene of Mycobacterium tuberculosis. Antimicrob Agents Chemother 1994; 38: 805-811.

13. Rossau R, Traore H, De Beenhouwer $\mathrm{H}$ et al. Evaluation of the INNO-LiPA Rif. TB Assay, a reverse hybridisation assay for the simultaneous detection of Mycobacterium tuberculosis complex and its resistance to rifampicin. Antimicrob Agents Chemother 1997; 41: 2093-2098.

14. Telenti A, Imboden P, Marchesi F et al. Detection of rifampicin-resistance mutations in Mycobacterium tuberculosis. Lancet 1993; 341: 647-650.

15. Scorpio A, Zhang Y. Mutations in pncA, a gene encoding pyrazinamidase/nicotinamidase; cause resistance to the antituberculous drug pyrazinamide in tubercle bacillus. Nat Med 1996; 2: 662-667.

16. Hirano K, Takahashi M, Kazumi Y, Fukasawa Y, Abe C. Mutation in pncA is a major mechanism of pyrazinamide resistance in Mycobacterium tuberculosis. Tubercle Lung Dis 1998; 78: $117-122$

17. Scorpio A, Lindholm-Levy P, Heifets et al. Characterization of pncA mutations in pyrazinamide-resistant Mycobacterium tuberculosis. Antimicrob Agents Chemother 1997; 41: $540-543$.

18. Screevatsan S, Pan X, Zhang Y, Kreiswirth BN, Musser JM. Mutations associated with pyrazinamide resistance in pncA of Mycobacterium tuberculosis complex organisms. Antimicrob Agents Chemother 1997; 41: 636-640.

19. Tahaoğulu K, Kizkin O, Karagöz T, Tor M, Partal M, Sadoğulu T. High initial and acquired drug resistance in pulmonary tuberculosis in Turkey. Tubercle Lung Dis 1994; 75: 324-328. 
20. Breathnach AS, de Ruiter A, Holdsworth GMC et al. An outbreak of multi-drug-resistant tuberculosis in a London teaching hospital. J Hosp Infect 1998; 39: 111-117.

21. Brown TJ, Power EGM, French GL. Evaluation of three commercial detection systems for Mycobacterium tuberculosis where clinical diagnosis is difficult. J Clin Pathol 1999; 52: 193-197.

22. Nolte FS, Metchock. Mycobaterium. In Murray PR, Baron EJ, Pfaller MA, Tenover FC, Yolken RH (eds) Manual of clinical microbiology, 6th edn. Washington, DC, ASM Press. 1995.

23. Wayne LG. Simple pyrazinamidase and urease tests for the routine identification of mycobacteria. Am Rev Respir Dis 1974; 109: 147-151.

24. van Embden JDA, Cave MD, Crawford JT et al. Strain identification of Mycobacterium tuberculosis by DNA fingerprinting: recommendations for standardized methodology. J Clin Microbiol 1993; 31: 406-409.

25. Shawar RM, el-Zaatari FAK, Nataraj A, Clarridge JE. Detection of Mycobacterium tuberculosis in clinical samples by two-step polymerase chain reaction and nonisotopic hybridisation methods. J Clin Microbiol 1993; 31: 61-65.
26. Brown TJ, Ison CA. Non-radioactive ribotyping of Haemophilus ducreyi using a digoxigenin labelled cDNA probe. Epidemiol Infect 1993; 110: 289-295.

27. Hewlett D, Horn DL, Alfalla C. Drug-resistant tuberculosis: inconsistent results of pyrazinamide susceptibility testing. JAMA 1995; 273: 916-917.

28. Salfinger M. Drug-resistant tuberculosis: inconsistent results of pyrazinamide susceptibility testing. JAMA 1995; 273: 914.

29. Trivedi SS, Desai SG. Pyrazinamidase activity of Mycobacter ium tuberculosis - a test of sensitivity to pyrazinamide. Tubercle 1987; 68: 221-224.

30. Kim B-J, Kim S-Y, Park B-H et al. Mutations in the rpoB gene of Mycobacterium tuberculosis that interfere with PCR-single-strand conformation polymorphism analysis for rifampicin susceptibility testing. J Clin Microbiol 1997; 35: $492-494$

31. Niemann S, Richter E, Rüsch-Gerdes S. Stability of Mycobacterium tuberculosis IS6110 restriction fragment length polymorphism patterns and spoligotypes determined by analyzing serial isolates from patients with drug-resistant tuberculosis. J Clin Microbiol 1999; 37: 409-412. 